\title{
Effects of dense code-switching on executive control
}

Article

Accepted Version

Hofweber, J., Marinis, T. and Treffers-Daller, J. (2016) Effects of dense code-switching on executive control. Linguistic Approaches to Bilingualism, 6 (5). pp. 648-668. ISSN 18799272 doi: https://doi.org/10.1075/lab.15052.hof Available at https://centaur.reading.ac.uk/65733/

It is advisable to refer to the publisher's version if you intend to cite from the work. See Guidance on citing.

To link to this article DOI: http://dx.doi.org/10.1075/lab.15052.hof

Publisher: John Benjamins

All outputs in CentAUR are protected by Intellectual Property Rights law, including copyright law. Copyright and IPR is retained by the creators or other copyright holders. Terms and conditions for use of this material are defined in the End User Agreement.

\section{www.reading.ac.uk/centaur}

\section{CentAUR}

Central Archive at the University of Reading

Reading's research outputs online 
Effects of Dense Code-switching on Executive Control

Running Head: Effects of Dense Code-switching 


\begin{abstract}
Bilingualism is reported to re-structure executive control networks, but it remains unknown which aspects of the bilingual experience cause this modulation. This study explores the impact of three code-switching types on executive functions: (1) alternation of languages, (2) insertion of lexicon of one language into grammar of another, (3) dense code-switching with co-activation of lexicon and grammar. Current models hypothesise that they challenge different aspects of the executive system because they vary in the extent and scope of language separation. Two groups of German-English bilinguals differing in dense code-switching frequency participated in a flanker task under conditions varying in degree of trial-mixing and resulting demands to conflict-monitoring. Bilinguals engaging in more dense code-switching showed inhibitory advantages in the condition requiring most conflict-monitoring. Moreover, dense code-switching frequency correlated positively with monitoring skills. This suggests that the management of co-activated languages during dense code-switching engages conflict-monitoring and that the consolidation processes taking place within coactivated linguistic systems involve local inhibition. Code-switching types requiring greater degrees of language separation may involve more global forms of inhibition. This study shows that dense code-switching is a key experience shaping bilinguals' executive functioning and highlights the importance of controlling for participants' code-switching habits in bilingualism research.
\end{abstract}




\section{Introduction}

Several recent studies have reported executive control (EC) advantages for bilinguals compared to monolinguals (Bialystok \& Luk, 2012). These are attributed to enhanced inhibitory control arising from bilinguals' need to continuously inhibit one of their languages (Green, 1998). However, several studies failed to replicate bilingual advantages at inhibition, leading some authors to question the robustness of the bilingual advantage (Paap \& Greenberg, 2013). To address this issue it is necessary to explain the observed variability by pin-pointing which bilingual experiences modulate which EC mechanisms. Rather than comparing bilinguals to monolinguals, this study addresses in detail variables differentiating bilinguals. We focus exclusively on adult bilinguals selected to differ on code-switching, a key experience proposed to modulate mechanisms leading to enhanced EC (Costa et al., 2009).

Code-switching is defined as the mixing of languages for socio-pragmatic optimization purposes (Bhatt \& Bolonyai, 2011). Code-switching involves two key processes: inhibitory control and conflict-monitoring of co-activated languages (Bialystok et al., 2012; Costa et al., 2009). Given the complexity of bilingual language control mechanisms employed during code-switching, it would be surprising if frequent code-switching did not shape EC networks in some way. Nevertheless, to date very few studies have investigated the relationship between code-switching and EC. Moreover, although bilinguals have been shown to engage in qualitatively different code-switching types (Muysken, 2000) associated with different cognitive control processes (TreffersDaller, 2009; Green \& Wei, 2014), all existing studies only investigated overall codeswitching frequency. This study measured for the first time not only the quantity of code-switching but also the code-switching types used by participants. To avoid 
confounding between-group effects it is also crucial to isolate key variables of bilingualism within relatively homogeneous participant groups. In this study all participants share the same German-English language combination and have been carefully matched for age, Socio-Economic Status (SES) and non-verbal IQ. If codeswitching is connected to differential EC performance, this finding could be extrapolated to the level of the monolingual-bilingual comparison and help explain previously observed inconsistencies.

\section{Code-switching and its impact on executive functions}

The interest in code-switching and executive functions goes back to Costa et al. (2009) reviewing 25 studies of bilingualism and executive functions using Stroop, Simon or Flanker tasks. These tasks measure inhibition in the so-called conflict effect, comparing RTs in incongruent trials taxing inhibition to RTs in congruent trials that do not require inhibition. Congruent and incongruent trials can either be administered in blocked or in mixed experiments. The review revealed that inhibitory advantages are mainly found in mixed designs requiring participants to switch between trial types within the same block. This suggests that bilingual advantages may not be attributable to inhibition alone, but to enhanced conflict-monitoring. Conflict-monitoring is a cognitive control mechanism involving the management of co-activated conflicting task-schemata allowing for flexible and rapid adaptation to changes in behavioural goals or task requirements (Botvinick et al., 2001). It is thus reminiscent of the notion of mental flexibility initially proposed to be at the core of bilingual performance advantages (Peal \& Lambert in Bialystok et al., 2012).

Costa et al. (2009) investigated this relationship between bilingualism, conflictmonitoring and inhibition by comparing bilingual Spanish-Catalan and monolingual 
Spanish participants' overall RTs and inhibitory skills in flanker tasks presented in low and high conflict-monitoring contexts. In the flanker task participants need to indicate the direction of a target arrow. Incongruent trials present distractor arrows that need to be inhibited. In the two low-monitoring conditions, the trial type split was $92 \%$ congruent to $8 \%$ incongruent and $92 \%$ incongruent to $8 \%$ congruent, whilst in the two high-monitoring conditions the split was $75 \%$ congruent to $25 \%$ incongruent and $50 \%$ congruent to $50 \%$ congruent, thus posing greater demands to conflict-monitoring. As predicted, bilingual advantages were confined to high-monitoring conditions. In these conditions, participants constantly need to be prepared to activate inhibitory mechanisms to solve forthcoming tasks, without knowing which trial type will be next (Bialystok et al., 2012). This mirrors the intricate interplay of inhibitory and monitoring processes during code-switching. Code-switching requires constant management of resources from both languages to react to and flexibly amend language choices to accommodate interlocutors. It thus employs cognitive control under high-monitoring conditions during language production and comprehension. This led Costa et al. (2009) to hypothesise that bilingual EC advantages may partially stem from code-switching practices. Neural evidence that similar frontal brain regions get activated during task and language switching adds further credibility to the hypothesis that code-switching trains mental flexibility (Abutalebi \& Green, 2008).

The impact of code-switching on executive functions has since been investigated in several studies, finding positive correlations between self-reported code-switching frequency and task-switching performance (Prior \& Gollan, 2011). Soveri et al. (2011) assessed Swedish-Finnish bilinguals' self-reported code-switching behaviour and administered task-switching tests to them. Crucially, mental flexibility was tested using 
two different measures: (1) switching cost representing RT or accuracy cost from switching between trial types within one block, and (2) mixing cost representing highlevel global monitoring processes managing co-activated task-schemata, calculated as the RT and accuracy difference between mixed and non-mixed blocks. Code-switching frequency predicted a reduced global mixing cost for accuracy, but there was no significant relationship with switching cost. This suggests that code-switching involves the continuous management of simultaneously co-activated languages rather than lowlevel switching processes (Soveri et al., 2011). In the present study we therefore focus on high-level processes managing task-coactivation, although we measure these in the monitoring rather than the mixing cost. Mixing involves switching between different task instructions, e.g. from sorting by colour to sorting by shape, whilst conflictmonitoring involves deactivation and re-activation of inhibitory schemata.

To date, most studies investigating code-switching and executive functions have focused on overall code-switching frequency without differentiating code-switching types. Within linguistic code-switching research, a rich literature has identified various types of code-switching differing in terms of processing, which has implications for the control processes assumed to be involved. Muysken (2000) identified three types of code-switching listed in order of decreasing language separation and increasing coactivation: (1) alternation of structurally independent stretches of two languages, (2) insertion of lexical items from one language into the grammar of another matrix language, and (3) congruent lexicalisation (here: dense code-switching) involving lexical and grammatical co-activation of both languages. Table 1 provides GermanEnglish examples of each type. The dense code-switching example "Wir haben FRIENDS gemacht mit'm SHOP OWNER." contains a calque of the English idiom "we 
made friends". Whilst this resembles an English matrix sentence filled with German and English lexemes, closer inspection reveals that the speaker follows German word order with an auxiliary verb (haben) in the second position and a past participle (gemacht) after the object (FRIENDS) followed by a mixed-language prepositional phrase (mit'm SHOP OWNER). This demonstrates that both grammar and lexicon of both languages are co-activated in dense code-switching.

\section{Table 1}

Based on the idea that greater language separation equates greater inhibitory control to suppress non-target varieties, Treffers-Daller $(1998,2009)$ proposed a continuum of inhibitory control involvement (Figure 1). During alternation, languages become temporarily suppressed implying relatively high inhibitory involvement. In insertion the lexicon of the non-matrix language is co-activated whilst its grammar remains inhibited, suggesting partial inhibition. Dense code-switching implies coactivation of grammar and lexicon with features being selected from either language. As there is little inhibition by language membership, dense code-switching practices inhibition least.

Figure 1

The Control Process Model of Code-switching CPM (Green \& Wei, 2014) provides a model of cognitive processes operating during code-switching. Whilst in monolingual modes competitive language schema coordination allows for the 
suppression of unintended languages, code-switching calls for cooperative language schema coordination. Two types of qualitatively different cooperative control modes are proposed: coupled control and open control mode. Insertion and alternation are governed by coupled control mode managing co-activated varieties through inhibition and language schema switching. In dense code-switching, an open control mode operates involving no discrimination by language membership. In terms of the interaction of code-switching with EC, the CPM predicts that alternation and insertion practice EC, whilst dense code-switching enhances EC minimally, if at all. Differential effects of code-switching on EC should thus be most salient when comparing bilinguals that densely code-switch to those that don't.

Conflict-monitoring as such is not explicitly discussed by either model. However, the notion of cooperative language schema implies the need to manage linguistic co-activation. Languages are most equally activated during dense codeswitching, so the open control mode should pose greatest demands to conflictmonitoring. It is therefore surprising that the authors predict only coupled control modes to enhance EC, whilst open control modes are supposed to engage EC less than monolingual modes (Green \& Wei, 2014: 8). This seeming contradiction can be resolved if we take apart the notion of EC and make separate predictions for inhibition and monitoring. During dense code-switching the flipside of not inhibiting languages is predicted to be increased practice at monitoring skills, so whilst dense code-switching may not involve inhibition (Treffers-Daller, 2009), it should enhance monitoring.

To provide a parsimonious account of control processes during code-switching it is necessary to explain how unintended interference is managed in the open control mode. In fact the very term open control is an oxymoron, as control can by definition 
never be completely open. Indeed the CPM acknowledges that dense code-switching is not random, but "grammatically appropriate" (Green \& Wei, 2014:16). If EC is involved in the cross-linguistic consolidation processes proposed to be involved in dense code-switching, then dense code-switching would have an enhancing effect on the relevant elements of the EC system.

To investigate the EC mechanisms involved in dense code-switching, we differentiate between global inhibition of whole language networks and local inhibition involving selection processes within co-activated networks through inhibition of specific representations within them (De Groot \& Christoffels, 2006; Guo, Liu, Misra \& Kroll, 2011). Although the CPM does not discuss global versus local inhibition explicitly, monolingual modes can be inferred to use global inhibition, whilst coupled control modes employ local inhibition (Green \& Wei, 2014: 15:18). To account for the management of unintended interference during open control modes, we suggest that dense code-switching employs some form of local inhibition operating within coactivated networks. Following the logic of Treffers-Daller's (2009) continuous approach, we hypothesise a continuum ranging from most global to most local inhibition (Table 2). In monolingual modes, languages get inhibited most globally, followed by alternational code-switching involving temporary global inhibition. Insertion implies global inhibition of the non-matrix grammar and local inhibition within co-activated lexical networks. Finally, dense code-switching employs local inhibition within co-activated grammatical and lexical networks. The less global inhibition operates in a given code-switching mode, the greater is the involvement of monitoring of co-activated languages in combination with local inhibition. Dense code- 
switching is predicted to engage local inhibition under circumstances requiring monitoring of co-activated language schemata, i.e. high-monitoring conditions.

Table 2

\section{The present study}

The present study investigates whether EC is modulated by the type of code-switching. To address this question, the ideal research design would involve three bilingual groups of the same language pair engaging exclusively in each respective code-switching type. In reality, most code-switchers engage in all three types to some extent. The emergence of code-switching patterns depends on bilinguals' sociolinguistic environment. All bilinguals engage in insertion and alternation to some extent. In contrast, dense codeswitching predominantly occurs in established bilingual communities with several generations of language contact (Muysken, 2000). In this study, the German-English language pair was kept constant to avoid variation due to language typology. Therefore, we identified two groups of German-English bilinguals differing in their extent of dense code-switching as a result of their sociolinguistic environments: (1) L1-German bilingual L2-users of English who are $1^{\text {st }}$ generation immigrants to the UK, (2) 5 th generation heritage speakers of German in South Africa.

Code-switching preference was measured in a frequency judgement task. To investigate EC performance, flanker tasks were presented in low, medium and highmonitoring contexts increasing in the degree of congruent-incongruent trial-switching (Costa et al., 2009). This manipulation enabled us to calculate the conflict effect 
measuring inhibition under conditions of differing degrees of conflict-monitoring, as well as the conflict-monitoring cost resulting from increased trial-switching and to relate participants' EC performance to their code-switching behaviour. The experimental design was guided by the following research questions:

1) Do $5^{\text {th }}$ generation bilinguals differ from $1^{\text {st }}$ generation bilinguals in their frequency of use of different code-switching types? This tested Muysken's (2000) hypothesis that bilinguals exposed to sociolinguistic environments of several generations of language contact will display a greater tendency to densely code-switch than bilinguals in recent immigration contexts. The two groups were not predicted to differ in their usage of insertion and alternation.

2) Do the two groups differ in their EC performance? If $5^{\text {th }}$ generation bilinguals engage in more dense code-switching, we predict advantages at processes assumed to be involved in dense code-switching, namely local inhibition under high-monitoring conditions, as well as conflict-monitoring.

3) Is there a correlation between participants' EC performance and their dense codeswitching frequency? Dense code-switching should correlate positively with inhibitory performance under high-monitoring conditions and with monitoring skills.

To summarize, dense code-switching is predicted not to lead to global inhibitory advantages (Treffers-Daller, 2009; Green \& Wei, 2014), but may enhance local inhibition under high-monitoring circumstances, as well as overall conflict-monitoring skills challenged by language schema coordination (Green \& Wei, 2014).

\section{Methods}

\subsection{Participants}


Two groups of 22 German-English bilinguals (mean age=39, $\mathrm{SD}=15.5$, right-handed, no known visual or mental impairments) were compared:

Group 1: $5^{\text {th }}$ generation heritage speakers of German in South Africa $(\mathrm{N}=11$, mean age $=39, \mathrm{SD}=16.1$ ). Their L1 home language was German and systematic exposure to English began after the age of 6 . These bilinguals live in communities with longstanding multilingual traditions and speak at least one additional local language, e.g., Afrikaans, Zulu, Setswana.

Group 2: $1^{\text {st }}$ generation German immigrants in the $\mathrm{UK}(\mathrm{N}=11$, mean age $=39, \mathrm{SD}=15.6)$. German was their L1 and exposure to English started after the age of 8. L2-immersion began after the age of 18. All speak additional school-taught languages.

The groups were carefully matched on age and non-verbal abilities (Table 3), as measured using Ravens Standard Progressive Matrices (Raven, Raven, \& Court, 1998) $\left[\mathrm{F}(1,22)=0.13, \mathrm{SME}=24.89, \mathrm{p}=0.72, \eta^{2}=0.01\right]$.

Table 3

They were also matched on SES and education. Both groups come from middle-class backgrounds and include 10 participants holding BA degrees and 1 with A-levels.

The LH-questionnaire (Li et al., 2013) was used to obtain the participants' perceptions of their language proficiencies. All bilinguals rated their English proficiency as advanced with 6 out of 7 points and reported to be native-like German speakers. . This may be surprising given the heritage speaker status of Group 1. What differentiates their context from other heritage speaker environments is the availability of schooling in the heritage language at primary and secondary level, resulting in them 
becoming literate in the home language. Therefore they did not undergo the language dominance shift associated with school entry in heritage speaker contexts.

\subsection{Tasks}

Two experimental tasks assessed participants' code-switching habits and executive functions: a frequency judgment and a flanker task. They were created using Psychopy 1.81 and were presented on a 13-inch-screen laptop.

\subsubsection{Frequency Judgment task}

Naturalistic code-switching primarily occurs in informal registers associated with high degrees of interlocutor familiarity, making it hard to be replicated in experimental settings (Gardner-Chloros, 2009). Frequency judgement tasks have been argued to be representative of cognitive embedding indicating language usage frequency (Backus, 2015). Participants were presented with 56 utterances containing 14 code-switches of each type: 1) insertion English into German, 2) insertion German into English, 3) alternation, and 4) dense code-switching (Table 1).

The stimuli were authentic utterances from existing German-English codeswitching corpora (Eppler, 2005; Clyne, 2003) and were classified using Deuchar, Muysken \& Wang's (2008) criteria. The utterances were presented in audio and visual format in pseudo-randomized order to avoid priming participants into particular codeswitching modes. Participants were instructed to imagine that they were having an informal conversation with a German-English bilingual friend and were asked to rate the frequency with which they would encounter utterances similar to the stimuli on a scale from "1"="never" to "7"="all the time". We asked about "frequency" instead of 
"acceptability" because code-switching is often stigmatised and the term "acceptability" could introduce an unintended attitudinal element.

\subsubsection{Flanker Task}

Inhibition was measured using the flanker task chosen for its relatively high degree of task purity due to the intuitiveness of its instructions, which reduces confounding working memory load, thus measuring inhibition more "purely" (Costa et al., 2008). Participants were presented with rows of 5 arrows and instructed to indicate the direction of the central arrow by a key press. In the congruent condition all arrows faced in the same direction; in the incongruent condition the arrows surrounding the target arrow faced in the opposite direction (Figure 2).

Figure 2

The incongruent condition required participants to use inhibition to suppress the distractor arrows and yields higher RTs, which is attributed to an increase in inhibitory cognitive load. Inhibition is measured in the conflict effect calculated by subtracting RTs in the congruent from those in the incongruent condition. A smaller conflict effect indicates greater inhibitory skills. Each trial started with a fixation cross for $200 \mathrm{ms,}$ followed by the $1000 \mathrm{~ms}$ stimulus presentation with a $1500 \mathrm{~ms}$ response time. Trial intervals were jittered (Figure 3).

Figure 3 
The task started with 6 practice trials, followed by three blocks of 96 trials. The blocks differed in the proportion of congruent-incongruent trial-switching and resulting load to conflict-monitoring: Block 1 (low-monitoring): 92\% congruent/8\% incongruent trials; Block 2 (high-monitoring): 50\% congruent/50\% incongruent trials; Block 3 (medium-monitoring): $75 \%$ congruent $/ 25 \%$ incongruent trials. To avoid practice effects, blocks were not presented in order of increasing demands to monitoring skills.

The manipulation of the proportion of congruent/incongruent trials allows for the calculation of the conflict effect under "high-monitoring" conditions, requiring increased levels of mental flexibility, as well as in "low-monitoring contexts" requiring less conflict-monitoring. It also allows for the calculation of a monitoring cost calculated as the difference between overall RTs in the most mixed 50-50 block and in the least mixed 92-8 block. The smaller the monitoring cost, the better participants are at conflict-monitoring.

\section{Results}

\subsection{Frequency judgment task}

None of the code-switching types received a mean rating of "1"="never", demonstrating that bilinguals engage in all types of code-switching. Figure 4 presents the frequency judgments for the four code-switching types in the two participant groups.

Figure 4

To address group differences in the frequency judgment of the code switching types, a between-subjects multivariate ANOVA was conducted. This revealed no 
significant differences between the groups on the frequency scores for insertion English into German $\left[F(1,20)=0.11, \mathrm{MSE}=0.255 \mathrm{p}=0.74, \eta^{2}=0.01\right]$, insertion German into English $\left[F(1,20)=0.47, \mathrm{MSE}=0.51, \mathrm{p}=0.50, \eta^{2}=0.02\right]$ and alternation $[\mathrm{F}(1,20)=1.11$, MSE $\left.=1.73, p=0.31, \eta^{2}=0.05\right]$, but there was a significant between-group difference in dense code-switching $\left[F(1,20)=5.46, \mathrm{MSE}=6.04, \mathrm{p}=0.03, \eta^{2}=0.21\right]$. The GermanEnglish $5^{\text {th }}$ generation bilinguals rated dense code-switching frequency (mean=3.4, $\mathrm{SD}=1.2$ ) significantly more highly than the German-English $1^{\text {st }}$ generation bilinguals (mean=2.4, $\mathrm{SD}=0.9)$.

\subsection{EC task performance in the flanker task by group}

Mean number of errors by block ranged from 0.2 to 1.3 errors (out of 96 trials). A repeated-measures ANOVA was conducted with Group $\left(5^{\text {th }}\right.$ generation, $1^{\text {st }}$ generation $)$ as the between-subjects factor and Monitoring condition (low, medium, high) as the within-subjects factor. The analysis revealed no significant Group effect $\left[F(1,20)=0.607, \mathrm{MSE}=2.561, \mathrm{p}=0.445, \eta^{2}=0.029\right]$, Monitoring condition $\left[\mathrm{F}(1.4,28)=1.265, \mathrm{MSE}=3.272, \mathrm{p}=0.286, \eta^{2}=0.059\right)$ and no significant Group by Monitoring Interaction $\left(\mathrm{F}(1.4,28)=0.109, \mathrm{MSE}=0.282, \mathrm{p}=0.825, \eta^{2}=0.005\right)$. The very high accuracy indicates that participants performed at ceiling.

\subsubsection{Group comparison for monitoring cost}

Monitoring cost was calculated as the difference in overall RTs between the high- and low-monitoring conditions. Smaller monitoring cost indicates better conflict-monitoring skills. In line with predictions, the $5^{\text {th }}$ generation bilinguals engaging in more dense code-switching suffered a slightly reduced monitoring cost $(\mathrm{M}=4.7 \mathrm{~ms}, \mathrm{SD}=42.4)$ 
compared to the $1^{\text {st }}$ generation bilinguals $(\mathrm{M}=15.3 \mathrm{~ms}, \mathrm{SD}=46.4)$, but this difference did not reach statistical significance ANOVA $[\mathrm{F}(1,20)=0.31, \mathrm{MSE}=611.6, \mathrm{p}=0.58$, $\left.\eta^{2}=0.015\right]$

\subsubsection{Group comparison for the conflict effect}

Figure 5 presents the conflict effect measured as the difference between RTs in incongruent minus congruent trials.

Figure 5

To address between-group differences in the conflict effect, a repeated-measures ANOVA was conducted with Group $\left(5^{\text {th }}\right.$ generation, $1^{\text {st }}$ generation $)$ as the betweensubjects factor and Monitoring condition (low, medium, high) as the within-subjects factor. The RT analyses revealed a significant effect of Monitoring condition $\left[\mathrm{F}(1.4,28.5)=6.16, \mathrm{MSE}=5864.79, \mathrm{p}=0.011, \eta^{2}=0.24\right)$ and a significant Group by Monitoring Interaction $\left(F(1.4,28.5)=4.58, \mathrm{MSE}=4365.71, \mathrm{p}=0.029, \eta^{2}=0.19\right)$ indicating that the impact of Monitoring condition on conflict effect differed across the groups. When investigating this interaction, a between-subjects multivariate ANOVA showed no effect of Group in the low and medium monitoring conditions [low: $F(1,20)=0.38$, $\mathrm{MSE}=887.1, \mathrm{p}=0.55, \eta^{2}=0.02 ;$ medium: $\left.\mathrm{F}(1,20)=1.93, \mathrm{MSE}=2662, \mathrm{p}=0.18, \eta^{2}=0.09\right]$ indicating no between-group difference in inhibition when little trial-switching was required. However, in the high-monitoring condition the Group effect on conflict effect was significant $\left[F(1,20)=6.79, \mathrm{MSE}=5923.68, \mathrm{p}=0.017, \eta^{2}=0.25\right]$ indicating that the $5^{\text {th }}$ generation bilinguals who densely code-switch more experienced a smaller conflict 
effect $(47.56 \mathrm{~ms})$ than the bilinguals engaging in less dense code-switching $(80.36 \mathrm{~ms})$ in the condition posing greatest load to conflict-monitoring. This means that the group practicing most dense code-switching outperformed the other group at a type of inhibition that is recruited in situations challenging mental flexibility.

Finally, repeated-measures ANOVAs for each group separately comparing the three Monitoring conditions in each group showed no significant difference between the three Monitoring conditions in the $1^{\text {st }}$ generation bilinguals $[\mathrm{F}(2,20)=0.05, \mathrm{MSE}=44.87$, $\left.\mathrm{p}=0.96, \eta^{2}=0.004\right]$ in conflict effect, and a significant difference between the Monitoring conditions in the $5^{\text {th }}$ generation bilinguals $[F(1.3,20)=20.18$, $\left.\mathrm{MSE}=11159.52, \mathrm{p}=0.001, \eta^{2}=0.67\right]$. Pairwise comparisons corrected for multiple comparisons using Bonferroni correction [significance level=0.017] showed that the conflict effect was significantly smaller in the medium $(57.73 \mathrm{~ms})$ and high $(47.56 \mathrm{~ms})$ compared to the low (96.2ms) monitoring condition [medium vs. low:p=0.009; high vs. low:p=0.001].

\subsection{Correlations between code-switching and EC performance}

\subsubsection{Correlation between code-switching and conflict effect}

Based on the directional hypothesis that inhibition under high-monitoring circumstances would correlate positively with dense code-switching, one-tailed Pearson's correlations were conducted between the conflict effect in the three conditions and frequency scores to different code-switching types (Table 4). These showed no significant correlations between code-switching and conflict effect in any of the three monitoring conditions.

Table 4 


\subsubsection{Correlation between code-switching and monitoring cost}

Based on previous research (Prior \& Gollan, 2011; Soveri et al., 2011) our directional hypothesis predicted a negative correlation between code-switching and monitoring cost. One-tailed Pearson's correlations for all participants together addressed whether the different types of code-switching judgment scores correlate negatively with monitoring cost (Table 5).

Table 5

There was no correlation between monitoring cost and Insertion of English into German, Insertion of German into English or Alternation. In contrast, there was a significant negative correlation between dense code-switching scores and monitoring cost $\left[\mathrm{r}(20)=-0.368, \mathrm{p}<0.046, \mathrm{R}^{2}=0.135\right]$. In line with predictions, the more frequently participants indicated to densely code-switch, the better they performed at conflictmonitoring.

\section{Discussion}

This study explored three research questions. Firstly, we tested whether the prediction that dense code-switching occurs more frequently in bilingual communities with extended language contact holds true for our bilingual groups. Secondly, we compared the groups regarding their EC performance, predicting that the group engaging in more 
dense code-switching would show advantages at inhibition under high-monitoring conditions, as well as at conflict-monitoring. Thirdly, a potential relationship between code-switching scores and EC was investigated. In line with predictions, $5^{\text {th }}$ generation bilinguals reported greater frequency at dense code-switching than $1^{\text {st }}$ generation immigrants. The group engaging in more dense code-switching showed inhibitory advantages in the high-monitoring condition. There were no significant group differences for monitoring cost, but results went in the direction of predictions with dense code-switchers demonstrating a slightly reduced monitoring cost. Finally, codeswitching did not correlate directly with inhibitory control under any of the three monitoring conditions, but there was a negative correlation between monitoring cost and dense code-switching frequency.

The predictions regarding the occurrence of code-switching were confirmed. The two groups engaged equally frequently in insertion and alternation, but differed in terms of their dense code-switching frequency. With increasing language contact there is a shift towards dense code-switching. Therefore bilinguals from a sociolinguistic environment of several generations of language contact indicated greater frequency of dense code-switching than recent immigrants (Muysken, 2000). As a consequence, they practice the types of inhibition involved in insertion and alternation equally frequently, but the heritage speakers get enhanced practice at EC processes required for dense codeswitching.

In the group comparison, bilinguals engaging in most dense code-switching outperformed the other group at inhibition in the condition requiring most conflictmonitoring. This is in line with our prediction that dense code-switchers will excel under high-monitoring conditions because cognitive processes operating under high- 
monitoring conditions mirror those taking place during dense code-switching. Therefore, any advantages for dense code-switchers would occur in high-monitoring contexts. What is surprising is that the dense code-switchers did not outperform the other group (significantly) at monitoring cost. Although the advantages only occur under high-monitoring conditions, they are inhibitory in nature. This could be argued to contradict existing models, which do not predict inhibitory advantages for dense codeswitchers. However, this contradiction can be resolved if we assume that the morphosyntactic control processes activated during dense code-switching (Green \& Wei, 2014) involve some form of inhibitory control processes. Importantly, it can therefore be hypothesised that monitoring co-activated languages during dense codeswitching does recruit inhibition, but of a highly local, not a global type. After all, linguistic interference needs to be managed and full co-activation calls for local forms of interference suppression. If dense code-switching draws upon more local forms of inhibition and insertion and alternation employ more global inhibition, then this would be in line with Green \& Wei (2014) postulating qualitative differences between open and coupled control modes. It also suggests that it is necessary to refine existing models by adding the dimension of global versus local inhibition. To pursue this issue, future bilingualism research should introduce tasks teasing apart global and local inhibition (Bialystok, 2010).

The observed effects could also be explained by the fact that dense codeswitching differs in scope, as there is not only lexical, but also grammatical coactivation (Green \& Wei, 2014:13). This could mean that qualitatively different processes are involved in managing lexical and grammatical competition. Only structural co-activation might involve temporal control of morphosyntax (Green \& 
Abutalebi, 2013). All bilinguals need to monitor language choice but not all types of bilinguals perform equally well at tasks placing demands on the EC system. Training at dealing with competing structural schemata may give dense code-switchers certain advantages over other bilinguals. Our findings can thus be reconciled with the CPM, if we assume that dense code-switching places particular demands on the temporal control of morphosyntax (Green \& Wei, 2014) and that the consolidation of competing grammatical structures draws upon EC.

Another reason why the observed advantage is inhibitory in nature is that inhibition and conflict-monitoring are not mutually exclusive, but interrelated (Costa et al., 2009; Morales et al., 2015). In the flanker task their interrelatedness is inevitable because conflict-monitoring involves constant preparedness to activate and de-activate inhibition to solve forth-coming trials. Similarly, dense code-switching calls for constant readiness to dynamically switch from suppressing cross-linguistic influence to lifting that suppression, thus monitoring the selection and combination of co-activated grammatical and lexical items in order to fulfil the communicative task at hand. This interplay of conflict-monitoring and inhibition could explain why bilinguals engaging in more dense code-switching excel at the task element measuring the combined effort of monitoring and inhibitory skills, i.e. specific inhibitory advantages limited to highmonitoring circumstances.

A further observation was that significant differences in inhibition by monitoring context only occurred amongst the bilinguals engaging in most dense codeswitching. $1^{\text {st }}$ generation bilinguals performed equally at inhibition across all conditions. By contrast, $5^{\text {th }}$ generation bilinguals who were used to dense codeswitching experienced reduced inhibitory costs in high-monitoring contexts; conditions 
challenging monitoring are less effortful for them. It is thus possible that highmonitoring local inhibitory processes akin to those recruited during dense codeswitching are their default control mode. This is in line with Green \& Wei (2014) proposing that neural networks involved in bilingual language control adapt to catering for control modes regularly employed. If dense code-switchers regularly activate inhibition under high-monitoring conditions, then these processes become highly automatized and would indeed be predicted to be less effortful.

The inhibitory advantage for our dense code-switchers under high-monitoring conditions is consistent with Costa et al.'s (2009) findings detecting differences between bilinguals and monolinguals in high-monitoring contexts. This is not surprising because it is possible that their Catalan-Spanish bilinguals were dense code-switchers. Catalonia houses the sociolinguistic environments in which dense code-switching is predicted to flourish (Muysken, 2000), i.e. communities with long-standing language contact between typologically closely related languages. If dense code-switching enhances the type of inhibition activated during conflict-monitoring, then its effects should be observable both when comparing dense code-switchers to other bilinguals (this study) and when comparing them to monolinguals (Costa et al., 2009).

This raises the question to which extent our findings contribute to explaining previously observed null effects in bilingualism research. It is conceivable that some null effects may have been due to a lack of controlling for bilingual participants' language usage patterns, such as code-switching. An absence of differential effects may also be attributable to the task type. The group differences observed in this study support Costa et al.'s (2009) hypothesis that bilingual advantages in EC tasks are more likely to occur under conditions requiring trial-switching, when the bilingual 
participants are frequent and dense code-switchers. It is thus crucial to control for both bilingual language usage variables and match these with relevant task types testing the specific EC functions predicted to be modulated by the speech pattern under investigation.

Finally, correlational analyses reveal a negative relationship between monitoring cost and dense code-switching scores. The more frequently bilinguals engaged in dense code-switching, the better they performed at conflict-monitoring, i.e. the mental flexibility of rapidly de- and re-activating inhibitory schemata. This indicates that dense code-switching enhances conflict-monitoring and mental flexibility. This is consistent with Soveri et al.'s (2011) finding of a negative correlation between codeswitching and mixing cost. Counter to predictions, we found no direct correlation between code-switching scores and inhibition. A possible explanation is that differential effects of code-switching type on inhibition may be too subtle to be detected within participants who engage in all types of code-switching to some extent. Correlations between code-switching scores and inhibition could have been observed if we had had participants engaging in one code-switching type exclusively, but these bilinguals are rare in real life.

Our findings are novel and contribute to our understanding of the experiences modulating bilinguals' EC abilities. However, the sample size is small. To increase the robustness of these findings it is necessary to replicate them within larger samples. Moreover, socio-cultural factors and differences between German varieties may confound the results because the groups were located in different countries. This potential confound could be eliminated by looking at individual differences in codeswitching preferences within the same social network. Future research could thus 
explore the differential impact of code-switching types on EC functions within the same community.

\section{Conclusion}

This study explored the differential impact of code-switching types on EC, thus shedding light on the processes underlying bilingual language control. Bilinguals from communities with long-standing language contact engaging in more dense codeswitching display inhibitory advantages in flanker tasks under conditions posing greatest load to conflict-monitoring. This suggests that the intricate interplay of monitoring and local inhibition required to solve the task mirrors cognitive processes taking place during dense code-switching. The fact that dense code-switching scores correlate negatively with monitoring cost suggests a positive relationship between practice at dense code-switching and mental flexibility. This is in line with the Control Process Model of Code-switching (Green \& Wei, 2014) suggesting that dense codeswitching involves the management of co-activated language structures. It also suggests that the control of interference within temporal neural networks containing morphosyntactic representations draws upon local inhibitory executive functions. To conclude, this study has shown for the first time that a specific type of code-switching, dense code-switching, is a key linguistic experience shaping bilinguals' executive functioning and enhances mental flexibility. 


\section{References}

Abutalebi, J., Green, D.W., (2008). Control mechanisms in bilingual language production: Neural evidence from language switching studies. Language and Cognitive Processes, 23, 557-582.

Backus, A. (2015). A usage-based approach to codeswitching: The need for reconciling structure and function. In: G. Stell, K. Yapko eds. (Eds). Code-switching at the crossroads between structural and sociolinguistic perspectives. Berlin: Mouton de Gruyter.

Bhatt, R., Bolonyai, A. (2011). Code-switching and the optimal grammar of bilingual language use. Bilingualism: Language and Cognition, 14, 522-546.

Bialystok, E. (2009). Bilingualism: The good, the bad, and the indifferent. Bilingualism: Language and Cognition, 12, 3-11.

Bialystok, E. (2010). Global-Local and Trail-Making Tasks by Monolingual and Bilingual children: Beyond Inhibition. Developmental Psychology, 46, 93-105

Bialystok, E., Craik, F.I.G., Luk, G. (2012). Bilingualism: Consequences for Mind and Brain. Trends in Cognitive Science, 16, 240-250.

Botvinick, M., Carter, C.S., Braver, T.S., Barch, D.M., Cohen, J.D. (2001). Conflictmonitoring and Cognitive Control. Psychological Review, 108, 624-652.

Clyne, M. (2003). Dynamics of Language Change. Cambridge Approaches to Language Contact, Cambridge University Press.

Costa, A., Hernandez, M., Sebastian-Galles, N. (2008). Bilingualism aids conflict resolution: Evidence from the ANT task. Cognition, 106, 59-86. 
Costa, A., Hernandez, M., Costa-Faidella, J., Sebastian-Galles, N. (2009). On the bilingual advantage in conflict processing: Now you see it, now you don't. Cognition, 113, 135-149.

De Groot, A.M.B., Christoffels, I.K. (2006). Language Control in bilinguals:

Monolingual tasks and simultaneous interpreting. Bilingualism: Language and Cognition, 9, 189-201.

Deuchar, M., Muysken, P. \& Wang, S. (2008). Structured Variation in Codeswitching: Towards an Empirically Based Typology of Bilingual Speech Patterns. International Journal of Bilingual Education and Bilingualism, 10, 298-34. Eppler, E. (2005). The Syntax of German-English code-switching. PhD Thesis, UCL. Gardner-Chloros, P. (2009). Code-switching. Cambridge: Cambridge University Press. Green, D.W. (1998). Mental control of the bilingual lexico-semantic system. Bilingualism: Language and Cognition, 1, 67-81.

Green, D.W., Abutalebi, J. (2013). Language Control in Bilinguals: The Adaptive Control Hypothesis. Journal of Cognitive Psychology, 25, 515-530.

Green, D.W., Wei, L. (2014). A control process model of CS. Language, Cognition and Neuroscience, 24, 499-511.

Guo, T., Liu, H., Misra, M., Kroll, J.F. (2011). Local and global inhibition in bilingual word production: fMRI evidence from Chinese-English bilinguals. Neuroimage, $56,2300-2309$.

Li, P., Zhang, F., Tsai, E., Puls, B. (2013). Language history questionnaire (LHQ 2.0): A new dynamic web-based research tool. Bilingualism: Language and Cognition, $17,673-680$. 
Morales, J., Yudes, C., Gomez-Ariza, C.J., Teresa Bajo, M. (2015). Bilingualism modulates dual mechanisms of cognitive control: Evidence from ERPs. Neuropsychologia, 66, 157-169.

Muysken, P. (2000). Bilingual Speech: A Typology of Code-mixing. Cambridge: Cambridge University Press.

Paap, K.R., Greenberg, Z.I. (2013). There is no coherent evidence for a bilingual advantage in executive processing, Cognitive Psychology, 66, 232-258.

Prior, A., Gollan, T.H. (2011). Good language-switchers are good task-switchers: evidence from Spanish-English and Mandarin-English bilinguals. Journal of the International Neuropsychological Society, 17, 682-691.

Raven, J., Raven, J. C., \& Court, J. H. (1998). Manual for Raven's progressive matrices and vocabulary scales. Oxford: Oxford Psychologists Press.

Soveri, A., Rodriguez-Fornells, A., Laine, M. (2011). Is there a relationship between language switching and executive functions in bilingualism? Introducing a withingroup analysis approach. Frontiers in Psychology, 2, Article 183.

Treffers-Daller, J. (1998). The IC model and code-switching. Bilingualism: Language and Cognition, 1, 98-99.

Treffers-Daller, J. (2009). Code-switching and transfer: an exploration of similarities and differences. In: Bullock, B.E. and Almeida, J.T. (Eds). The Cambridge Handbook of Linguistic Codeswitching. Cambridge University Press, pp.58-74. 


\section{TABLES}

Table 1:Muysken's (2000) code-switching types (upper/lower case indicating different languages used)

\begin{tabular}{|l|l|}
\hline Code-switching type & Example \\
\hline Alternation & $\begin{array}{l}\text { Ich kann heute nicht kommen BECAUSE I'M ILL. } \\
\text { I can today not come BECAUSE I'M ILL. } \\
\text { I can't come today BECAUSE I'M ILL. }\end{array}$ \\
\hline Insertion & $\begin{array}{l}\text { Wir suchen noch VOLUNTEERS fuer das Projekt. } \\
\text { We search still VOLUNTEERS for the project. } \\
\text { We are still looking for VOLUNTEERS for the project. }\end{array}$ \\
\hline Insertion & $\begin{array}{l}\text { We didn't bring the right SCHUHWERK for hiking. } \\
\text { We didn't bring the right } \text { SHOES for hiking. } \\
\text { We didn't bring the right } \text { SHOES for hiking. }\end{array}$ \\
\hline Dense Code-switching & $\begin{array}{l}\text { Wir haben } \text { FRIENDS gemacht mit'm SHOP OWNER. } \\
\text { We have FRIENDS made with th' SHOP OWNER. }\end{array}$ \\
\hline
\end{tabular}

Table 2: Continuum of local and global inhibitory involvement and monitoring involvement

\begin{tabular}{|l|l|l|l|}
\hline dense code-switching & insertion & alternation & monolingual mode \\
\hline $\begin{array}{l}\text { Local inhibition of } \\
\text { lexical items }\end{array}$ & $\begin{array}{l}\text { Local inhibition of } \\
\text { non-matrix } \\
\text { lexical items } \\
\text { Local inhibition of } \\
\text { grammatical features }\end{array}$ & $\begin{array}{l}\text { Temporary } \\
\text { global inhibition of } \\
\text { non-matrix grammar }\end{array}$ & $\begin{array}{l}\text { Prolonged } \\
\text { Global inhibition }\end{array}$ \\
\hline \multicolumn{2}{|c|}{ most local } & most global \\
\hline \begin{tabular}{|l} 
most monitoring \\
\hline
\end{tabular}
\end{tabular}


Table 3:Participants' characteristics

\begin{tabular}{llccccc}
\hline & & $\begin{array}{c}\mathbf{5}^{\text {th }} \text { generation } \\
\text { bilinguals }\end{array}$ & $\begin{array}{c}\mathbf{1}^{\text {st }} \text { generation } \\
\text { bilinguals }\end{array}$ & F-value & df & p-value \\
\hline Age & Mean & 39 & 39 & 0.001 & 1,20 & 0.98 \\
& Range & $19-65$ & $22-70$ & & & \\
& SD & 15.5 & 16.1 & & & \\
Ravens & Mean & 108 & 110 & 0.128 & 1,20 & 0.72 \\
& Range & $85-130$ & $75-125$ & & & \\
& SD & 11.8 & 15.8 & & & \\
\hline
\end{tabular}


Table 4:Pearson's correlations between code-switching scores and conflict effect in high-monitoring condition

\begin{tabular}{|l|l|l|l|}
\hline Code-switching type & Pearson's r & Significance & Pearson's r \\
level & coefficient & 0.320 & {$[0.50 ;-0.33]$} \\
\hline Insertion E into G & 0.105 & 0.485 & {$[0.43 ;-0.41]$} \\
\hline Insertion G into E & 0.008 & 0.426 & {$[0.46 ;-0.39]$} \\
\hline Alternation & 0.042 & 0.495 & {$[0.42 ;-0.42]$} \\
\hline Dense code-switching & 0.003 & & \\
\hline
\end{tabular}


Table 5:Pearson's correlations between code-switching scores and monitoring cost

\begin{tabular}{|l|l|l|l|}
\hline Code-switching type & Pearson's r & Significance & Pearson's r \\
& coefficient & level & confidence interval \\
\hline Insertion E into G & -0.291 & 0.095 & {$[0.15 ;-0.63]$} \\
\hline Insertion G into E & -0.236 & 0.145 & {$[0.21 ;-0.60]$} \\
\hline Alternation & -0.262 & 0.120 & {$[0.18 ;-0.62]$} \\
\hline Dense & -0.368 & $0.046^{*}$ & {$[0.06 ;-0.68]$} \\
\hline
\end{tabular}




\section{FIGURES}

Figure 1:Treffers-Daller's inhibitory control continuum of code-switching (2009)

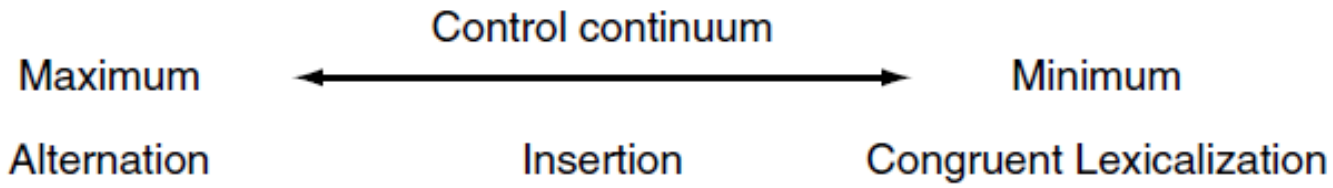

Figure 2:Congruent and incongruent stimuli

\begin{tabular}{|l|l|}
\hline$\longrightarrow \vec{\longrightarrow}$ & $\vec{\longrightarrow} \vec{\longrightarrow} \vec{\longrightarrow}$ \\
\hline congruent stimulus & incongruent stimulus \\
\hline
\end{tabular}

Figure 3:Flanker task presentation, individual trial

\begin{tabular}{|l|l|l|l|}
\hline \multicolumn{1}{|c|}{} & & & \\
& & & \\
\hline fixation cross & stimulus presentation & blank screen & trial interval \\
& response time & response time & jittered \\
& $1000 \mathrm{~ms}$ & $1500 \mathrm{~ms}$ & $100-3000 \mathrm{~ms}$ \\
\hline
\end{tabular}


Figure 4:Results of the Frequency Judgment Task

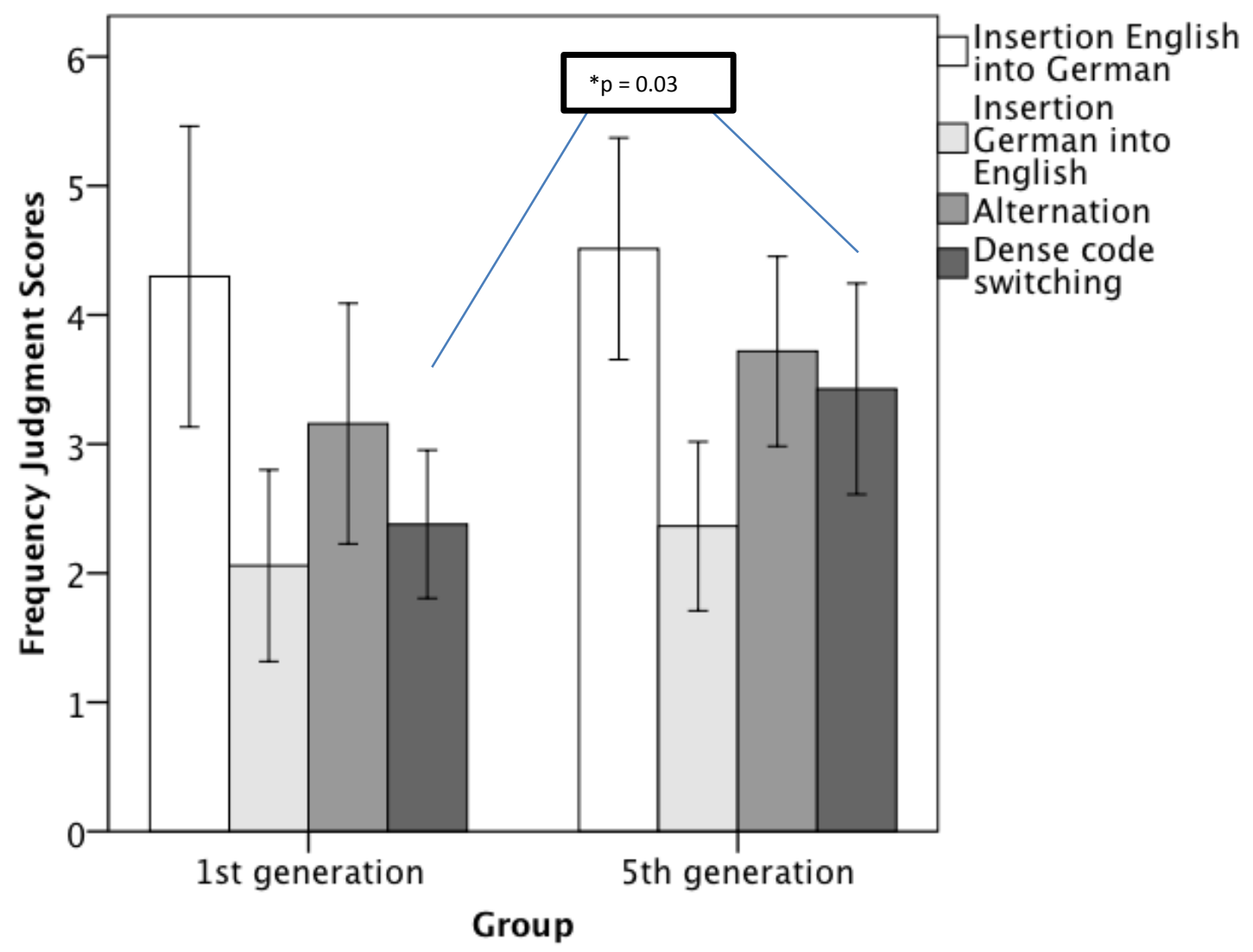

Error bars: $95 \% \mathrm{CI}$ 
Figure 5:Conflict Effect in high, medium, and low monitoring conditions of the Flanker Task

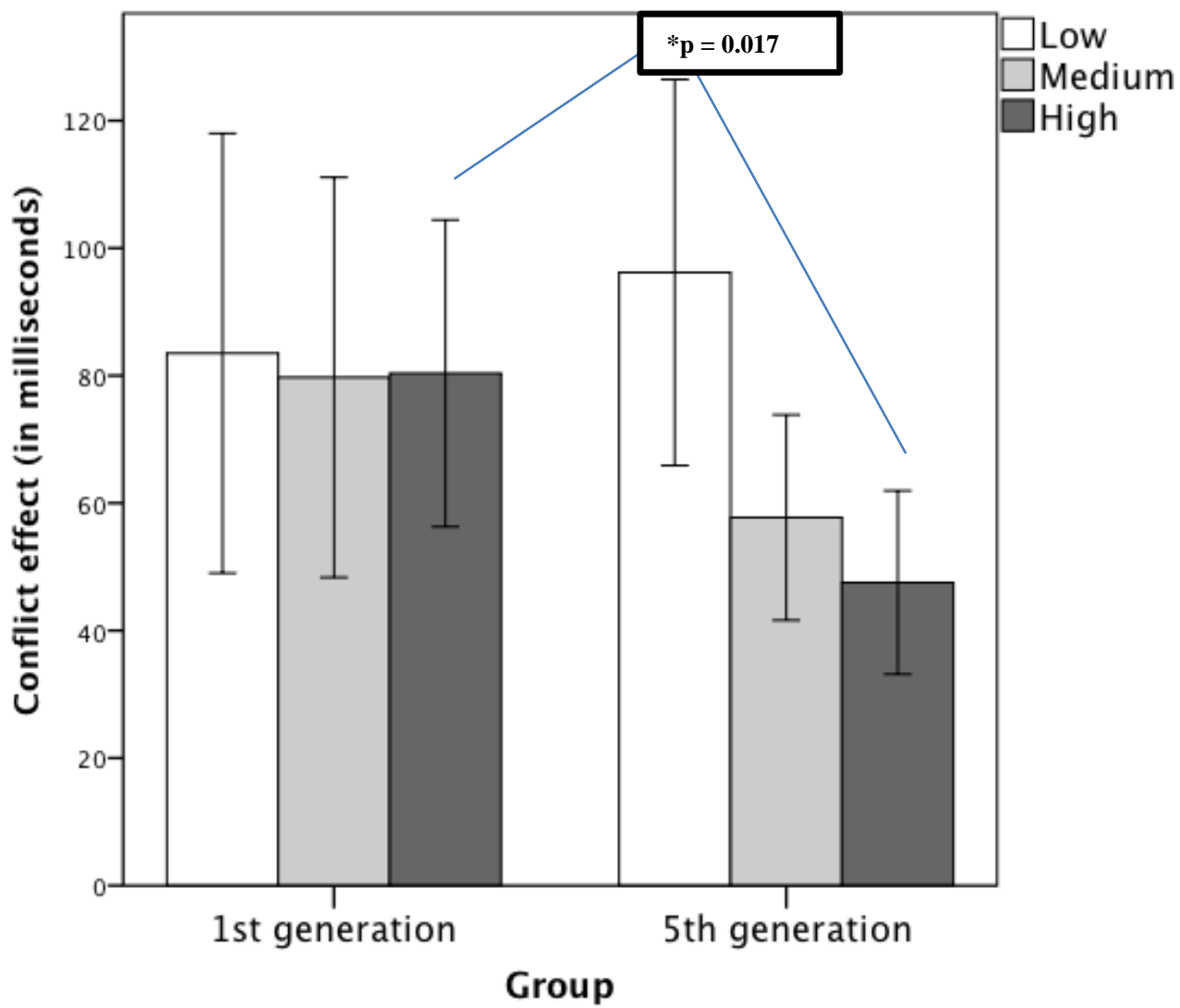

Error bars: $95 \% \mathrm{Cl}$ 